\title{
Impact of persistent substance misuse on 1-year outcome in first-episode psychosis
}

Aidan Turkington, Ciaran C. Mulholland, Teresa M. Rushe, Rick Anderson, Rosalind McCaul, Suzanne L. Barrett, Ruth S. Barr and Stephen J. Cooper

\section{Background}

Substance misuse is a common comorbid problem in people presenting with first-episode psychosis and is associated with a poor short-term outcome.

\begin{abstract}
Aims
The aim of this study is to examine differences in baseline characteristics and 1-year outcome between individuals with first-episode psychosis who have never misused substances, those who stop misusing substances after initial presentation and those who persistently misuse substances over the 1year assessment period.
\end{abstract}

\section{Method}

Patients were recruited to the Northern Ireland First Episode Psychosis Study $(n=272)$. Clinical assessments were

performed at baseline and at 1 year $(n=194)$ and data were collected from the case notes.

\section{Results}

Individuals with persistent substance misuse had more severe depression, more positive symptoms, poorer functional outcome and greater rates of relapse at 1 year than those who stopped and those who had never misused substances. There were no differences in outcome between people who had never misused substances and those who stopped misusing after presentation.

\section{Conclusions}

These results support assertive intervention targeted at comorbid substance misuse in individuals with first-episode psychosis.

\section{Declaration of interest}

None.
Rates of comorbid substance misuse in schizophrenia are up to five times higher than in the general population. ${ }^{1}$ Substance misuse is also a common comorbid problem in people presenting with first-episode psychosis, with rates of misuse varying between 11 and $62 \% .^{2-8}$ People with substance misuse present with their first episode of psychosis at a younger age, are more likely to be male ${ }^{3,5,9}$ and may present with less severe negative symptoms. ${ }^{10}$ Substance misuse has a negative impact on clinical outcome. ${ }^{4,6,11-13}$ In particular it has been shown that persistent substance misuse in the early course of illness is associated with increased risk of readmission and relapse of positive symptoms ${ }^{6}$ as well as poorer functional outcome. ${ }^{13}$ Individuals with persistent substance misuse have also been shown to have lower remission rates than those who stop misusing after presentation. ${ }^{4}$ The aim of this study was to determine the impact of persistent substance misuse on 1-year outcome in people with a first episode of psychosis. It was hypothesised that people who persistently misuse substances would have a worse outcome when compared with those who stop misusing after initial presentation and those who have never misused.

\section{Method}

\section{Participants}

Participants were recruited to the Northern Ireland First Episode Psychosis Study (NIFEPS) between 1 January 2003 and 31 December 2004. All incident cases of psychosis were included. Participants were aged 18-64 years, and were required to have lived in Northern Ireland for the 6 months prior to presentation. Individuals with previous psychotic diagnoses were excluded. Three hundred and seventy-five individuals were identified and 272 of them agreed to participate and were included in the study. Of these individuals 18 were identified through a 'leakage' study. These are individuals that were identified retrospectively through detailed examination of in-patient and out-patient records. They are not included in this analysis as their initial assessments were performed more than 1 year following presentation. One hundred and ninety-four people were followed up at 1 year. Baseline substance misuse information was not available for two individuals, and 1-year substance misuse information was not available in a further four people. These individuals were therefore excluded to leave 188 participants in the study. Of 60 people not followed up, 57 were uncontactable and 3 had died. All participants gave written, informed consent to be included in the study.

No specialised first-episode psychosis service exists in Northern Ireland. People were recruited from general psychiatric services from a number of health boards in Belfast City and the County Antrim area. Treatment was based on clinical need using locally available psychiatric and addiction services although the precise details of substance misuse treatment were not captured by this study.

\section{Clinical assessment and case-note analysis}

Face-to-face clinical assessment was performed at presentation and at 1 year. Assessments performed included the Positive and Negative Syndrome Scale (PANSS) ${ }^{14}$ and the Beck Depression Inventory $(\mathrm{BDI})^{15}$ at both initial assessment and 1 year. The Psychiatric and Personal History Schedule (PPHS) ${ }^{16}$ was completed at assessment and the Follow-up Psychiatric and Personal History Schedule (FU-PPHS) at 1 year. These collect a range of clinical and demographic information, including information on substance misuse, relapses, treatment received and medication adherence. The PPHS at baseline was also completed separately with next of kin where available $(n=97)$. Global Assessment of Functioning $(\mathrm{GAF})^{17}$ was assessed at 1 year only.

Case notes were analysed using the Operational Criteria checklist for Psychotic Illness (OPCRIT) ${ }^{18}$ to determine ICD-10 ${ }^{19}$ 
diagnosis at 1 year. A separate FU-PPHS was completed for each participant from information contained within the case notes.

Duration of untreated psychosis (DUP) was estimated from patient reports and from information collected using the PPHS from participants and relatives. Participants were divided into two groups; 'long' duration and 'short' duration based on DUP relative to the median value of 6 months.

Medication adherence was assessed at 1 year and was based on information contained within the FU-PPHS derived from clinical assessment and the case notes. Using the question on adherence contained within the FU-PPHS, poor adherence was defined as: 'lapses of 3 or more days more than once' or 'not taking any prescribed medication'. Participants were classed as adhering to their medication if they were not currently prescribed medication. Participants were classed as 'poor adherence' if this criterion was met in either clinical assessment or case notes.

\section{Substance misuse}

Information on substance use was derived from information contained in the PPHS completed during participant or informant interview at baseline and from the FU-PPHS completed during participant interview and from case notes at 1 year. At each time point, use of substances over the preceding year was recorded and categorised as: dependence, abuse, use, abstention or never used using DSM-IV criteria. This information was collected using a structured schedule (see Online supplement). 'Substance misuse' was defined accordingly as presence of either 'abuse' or 'dependence' and measured separately at each time point: baseline and 1 year.

\section{Outcomes}

Outcome measures were: GAF, BDI and PANSS factor scores and relapse at 1 year. The GAF, PANSS and BDI scores were obtained during face-to-face clinical assessment. Relapse was defined as either symptomatic relapse following a period of remission or no remission of symptoms within the first year. The appendix of the FU-PPHS contains an operational definition of 'relapse' incorporating the emergence or exacerbation of positive, negative or disorganised symptoms following a period of remission of at least 30 days. Similarly, 'remission' is also operationally defined as the absence of such symptoms for at least 30 days. Information on relapse was derived from information contained within the FU-PPHS obtained from case notes and supplemented by information from the FU-PPHS completed at clinical assessment.

\section{Statistical analysis}

Participants were divided into three groups for the purpose of statistical analysis: 'never' - people with no substance misuse at baseline or at 1 year; 'stopped' - people with substance misuse at baseline but not at 1 year; 'persistent' - people with substance misuse both at baseline and at 1 year. Two participants were found to be misusing substances at 1 year but not at baseline and were included in the persistent group.

Baseline characteristics for the three groups were compared using ANOVA for continuous variables and the Pearson chisquared test for categorical variables. Differences in baseline symptom scores were determined using ANCOVA (adjusting for age and gender). Differences in outcome were determined using ANOVA for GAF, PANSS and BDI scores, and the Pearson chisquared test for relapse. Results were then sequentially adjusted for confounding factors, namely age, gender and medication adherence, using ANCOVA. Baseline scores were also considered as confounding factors where they significantly differed between groups at baseline. Any confounding factors seen to influence outcome $(P<0.1)$ were retained within a final univariate ANOVA model. When considering group differences in PANSS and BDI scores at 1 year, the baseline symptom score was always included as a covariate in the final model.

These analyses were carried out on the group as a whole and repeated in the subgroup of participants with a diagnosis of schizophrenia-spectrum disorder.

All analyses were carried out using the Statistical Package for the Social Sciences version 14.0 for Windows XP.

\section{Results}

\section{Participants}

There were no significant differences in baseline characteristics between the participants who were not followed up $(n=60)$ and those who were followed up. Of the 194 participants followed up, information on substance misuse was available for 188. Of these, 103 (55\%) were diagnosed with a schizophrenia-spectrum disorder (schizophrenia and schizoaffective disorder) according to the OPCRIT at 1 year. Of the remainder, 24 individuals (13\%) were diagnosed with a depressive psychosis, 32 participants (17\%) with mania or bipolar affective disorder and 29 (15\%) with psychosis not otherwise specified. One person did not receive an OPCRIT diagnosis but was included in the study under 'psychosis not otherwise specified' based on the investigators' consensus clinical opinion of psychotic illness.

\section{Prevalence}

The rate of baseline substance misuse in the total sample was $43 \%$. The most misused substance was alcohol (33.0\%), followed by cannabis $(20.7 \%)$. At 1 year the rate of substance misuse had decreased in the total sample to $22.9 \%$. The rates of misuse for alcohol and cannabis at 1 year were $15.4 \%$ and $9.6 \%$ respectively. In the schizophrenia subgroup, rates of substance misuse were higher. The rate of baseline substance misuse was 50.5\%, falling to $24.3 \%$ at 1 year. Rates of misuse of alcohol and cannabis were $49.8 \%$ and $26.2 \%$ at baseline, falling to $17.5 \%$ and $10.7 \%$ respectively at 1 year.

\section{Baseline characteristics}

Comparisons of baseline characteristics by substance misuse group are displayed in Table 1 . There was a significant excess of males in the stopped $(P=0.02)$ and persistent $(P=0.001)$ groups compared with the never group, but there was no significant difference between the stopped and persistent groups. Age at presentation was significantly lower in both the stopped $(P<0.01)$ and persistent $(P<0.01)$ groups compared with the never group. Post hoc linear regression analysis with gender and baseline cannabis, alcohol and other substance misuse as independent variables showed a significant relationship between cannabis misuse and age at presentation $(B=-8.256$, s.e. $=2.27$, $\beta=0.274, P<0.001)$. Poor adherence to medication was more prevalent in the persistent group than in both the stopped $(P=0.01)$ and never $(P<0.001)$ groups. There was no significant difference in DUP between the three groups.

Regarding baseline scores, PANSS negative symptom scores varied significantly across the three substance misuse groups $(P=0.03)$. Group differences did not remain significant after adjusting for age and gender, with significantly higher PANSS negative scores in men than women (15.0 v. 11.9, $t=3.99$, $P<0.001)$. The PANSS positive symptom scores did not vary significantly across the three groups, but they became significant 
Table 1 Differences in baseline characteristics across substance misuse groups for all participants $(n=188)$

\begin{tabular}{|c|c|c|c|c|c|c|c|}
\hline & \multicolumn{4}{|c|}{ Substance misuse group } & \multirow[b]{2}{*}{$\begin{array}{c}\text { Test } \\
\text { statistic }\end{array}$} & \multirow[b]{2}{*}{$P$} & \multirow[b]{2}{*}{ Significant pairwise comparisons } \\
\hline & $n$ & $\begin{array}{l}\text { Never } \\
n=105\end{array}$ & $\begin{array}{l}\text { Stopped, } \\
n=40\end{array}$ & $\begin{array}{l}\text { Persistent, } \\
n=43\end{array}$ & & & \\
\hline \multicolumn{8}{|l|}{ Baseline characteristics } \\
\hline Gender, ratio male:female & 188 & $51: 49$ & $72: 28$ & $81: 19$ & $\chi^{2}=13.78$ & $<0.001$ & \multirow{4}{*}{$\begin{array}{l}\text { Persistent }>\text { Never }{ }^{* * *} \text {; Stopped }>\text { Never* } \\
\text { Persistent }<\text { Never }^{* *} \text {; Stopped }<\text { Never }^{* *}\end{array}$} \\
\hline Age at presentation, mean (s.d.) & 188 & $36.9(12.6)$ & $30.8(11.0)$ & $30.6(10.8)$ & $F=6.54$ & $<0.01$ & \\
\hline Long DUP, \% of substance & & & & & & & \\
\hline misuse group & 173 & 45.7 & 45.0 & 61.9 & $\chi^{2}=3.50$ & 0.17 & \\
\hline $\begin{array}{l}\text { Poor adherence, }{ }^{a} \% \text { of } \\
\text { substance misuse group }\end{array}$ & 181 & 17.5 & 24.3 & 48.8 & $\chi^{2}=15.01$ & $<0.001$ & Persistent $>$ Never $* \star *$; Persistent $>$ Stopped $*$ \\
\hline \multicolumn{8}{|l|}{ Baseline scores } \\
\hline PANSS general, ${ }^{\mathrm{b}}$ mean (s.e) & 185 & $29.88(0.78)$ & $28.10(1.39)$ & $30.62(1.54)$ & $F=0.86$ & 0.42 & \multirow{3}{*}{ Stopped > Never*; Stopped > Persistent* } \\
\hline PANSS positive, ${ }^{\mathrm{b}}$ mean (s.e) & 185 & $16.78(0.58)$ & $19.88(1.04)$ & $16.52(1.14)$ & $F=3.70$ & 0.03 & \\
\hline PANSS negative, ${ }^{\mathrm{b}}$ mean (s.e) & 185 & $13.22(0.50)$ & $12.95(0.89)$ & $15.26(0.98)$ & $F=1.96$ & 0.14 & \\
\hline $\mathrm{BDI},{ }^{\mathrm{b}}$ mean $(\mathrm{s} . \mathrm{e})$ & 169 & $17.59(1.26)$ & $15.37(2.36)$ & $24.34(2.71)$ & $F=3.41$ & 0.04 & Persistent $>$ Never*; Persistent $>$ Stopped* \\
\hline $\begin{array}{l}\text { DUP, duration of untreated psychosis; PAl } \\
\text { a. Measured at } 1 \text { year. } \\
\text { b. Adjusted for age, gender. } \\
\star P^{*}<0.05 \text {, }{ }^{* * P} P<0.01,{ }^{* \star \star P} P<0.001 \text {. }\end{array}$ & SS, P & ive and $\mathrm{Ne \xi}$ & yndrome & BDI, Beck & ssion In! & & \\
\hline
\end{tabular}

after adjustment, with significantly higher positive symptoms in the stopped group compared with the persistent $(P=0.03)$ and never $(P=0.01)$ groups. Baseline BDI scores varied significantly between the three groups both before and after adjustment, with significantly higher scores in the persistent group compared with both the stopped $(P=0.01)$ and never $(P=0.03)$ groups.

The results for the total sample were generally mirrored in the schizophrenia subgroup (Table 2).

\section{One-year outcome}

Unadjusted outcomes for the whole sample showed a significant $(P<0.05)$ effect of substance misuse group for each outcome measure (GAF scores, PANSS scores and relapse rate). The relapse rate was $34 \%$ (64 of 187 participants) and also varied significantly between substance misuse groups. Adjusted outcomes for the whole sample of participants are displayed in Table 3. Differences between substance misuse groups in GAF total $(P<0.01)$, GAF symptoms $(P<0.01)$ and GAF disability $(P<0.01)$ all remained significant after adjustment. For both GAF total and GAF disability there was no significant difference between the stopped and never groups, but the outcome in the persistent group was significantly worse than both. A significant interaction was seen between substance misuse and gender in GAF total and GAF disability, with women scoring lower than men in the persistent group but higher than men in the stopped and never groups. Of the PANSS subscales, only positive symptoms remained significant after adjustment. The persistent group $(P<0.01)$ and the never group $(P=0.03)$ both showed more severe positive symptoms at 1 year than the stopped group. Beck Depression Inventory scores also remained significantly different between the three groups. The persistent group showed more severe depressive symptoms than both the stopped $(P=0.03)$ and never $(P=0.02)$ groups. There was no difference in 1-year BDI score between the never and stopped groups. Adjusted relapse rates in the persistent group were significantly higher than in the stopped $(56.3 \%$ v. $32.9 \%$, $P=0.04)$ and never $(56.3 \%$ v. $35.6 \%, P=0.02)$ groups. There was no significant difference in relapse between participants who never used substances and those who stopped at 1 year.

Unadjusted outcomes for the schizophrenia subgroup displayed a similar pattern with a significant $(P<0.05)$ effect of substance misuse group for each outcome measure except GAF

\begin{tabular}{|c|c|c|c|c|c|c|c|}
\hline & \multicolumn{4}{|c|}{ Substance misuse group } & \multirow[b]{2}{*}{$\begin{array}{c}\text { Test } \\
\text { statistic }\end{array}$} & \multirow[b]{2}{*}{$P$} & \multirow[b]{2}{*}{ Significant pairwise comparisons } \\
\hline & $n$ & $\begin{array}{c}\text { Never, } \\
n=50\end{array}$ & $\begin{array}{c}\text { Stopped, } \\
n=28\end{array}$ & $\begin{array}{c}\text { Persistent, } \\
n=25\end{array}$ & & & \\
\hline \multicolumn{8}{|l|}{ Baseline characteristics } \\
\hline Gender, ratio male:female & 103 & $56: 54$ & $71: 29$ & $84: 16$ & $\chi^{2}=6.25$ & 0.04 & Persistent $>$ Never* \\
\hline $\begin{array}{l}\text { Age at presentation, mean (s.d.) } \\
\text { Long DUP, \% of substance }\end{array}$ & 103 & $36.0(13.7)$ & $28.0(7.9)$ & $26.4(9.3)$ & $F=7.70$ & $<0.001$ & Persistent $<$ Never $^{* * *}$; Stopped $<$ Never $^{* *}$ \\
\hline misuse group & 103 & 56.0 & 39.3 & 72.0 & $\chi^{2}=5.74$ & 0.06 & \\
\hline $\begin{array}{l}\text { Poor adherence, }{ }^{a} \% \text { of } \\
\text { substance misuse group }\end{array}$ & 98 & 26.5 & 20.0 & 58.3 & $\chi^{2}=9.85$ & $<0.01$ & Persistent $>$ Never**; Persistent $>$ Stopped** \\
\hline \multicolumn{8}{|l|}{ Baseline scores } \\
\hline PANSS general, ${ }^{\mathrm{b}}$ mean (s.e) & 102 & $31.07(1.20)$ & $28.96(1.69)$ & $29.94(1.20)$ & $F=0.51$ & 0.60 & \\
\hline PANSS positive, ${ }^{\mathrm{b}}$ mean (s.e) & 102 & $17.37(0.87)$ & $21.18(1.22)$ & $17.66(1.59)$ & $F=3.35$ & 0.04 & Stopped > Never* \\
\hline PANSS negative, ${ }^{b}$ mean (s.e) & 102 & $14.10(0.77)$ & $14.76(1.09)$ & $15.97(1.42)$ & $F=0.67$ & 0.52 & \\
\hline $\mathrm{BDI}^{\mathrm{b}}{ }^{\mathrm{m}}$ mean $(\mathrm{s} . \mathrm{e})$ & 95 & $19.38(1.89)$ & $14.93(2.85)$ & $28.26(3.34)$ & $F=4.72$ & 0.01 & Persistent $>$ Never*; Persistent $>$ Stopped** \\
\hline
\end{tabular}




\begin{tabular}{|c|c|c|c|c|c|c|c|}
\hline \multirow[b]{2}{*}{ Adjusted 1-year score } & \multicolumn{4}{|c|}{ Substance misuse group } & \multirow[b]{2}{*}{$F$} & \multirow[b]{2}{*}{$P$} & \multirow[b]{2}{*}{ Significant pairwise comparisons } \\
\hline & $n$ & Never & Stopped & Persistent & & & \\
\hline \multicolumn{8}{|l|}{ GAF, mean (s.e.) } \\
\hline GAF total ${ }^{a}$ & 171 & $56.58(1.78)$ & $61.29(2.78)$ & $49.16(2.66)$ & 5.13 & $<0.01$ & \multirow{3}{*}{$\begin{array}{l}\text { Persistent }<\text { Stopped } * * ; \\
\text { Persistent }<\text { Stopped } \text { St }^{* *} \text { Stent }<\text { Never }^{*} \\
\text { Persistent }<\text { Stopped }{ }^{* * *} \text {; Persistent }<\text { Never }^{*}\end{array}$} \\
\hline GAF symptoms ${ }^{b}$ & 171 & $58.24(1.82)$ & $64.46(2.62)$ & $52.74(2.72)$ & 5.69 & $<0.01$ & \\
\hline GAF disability ${ }^{a}$ & 171 & $57.09(1.82)$ & $62.96(2.77)$ & $50.11(2.65)$ & 5.64 & $<0.01$ & \\
\hline \multicolumn{8}{|l|}{ PANNS, mean (s.e.) } \\
\hline PANNS negative ${ }^{c}$ & 166 & $12.73(0.37)$ & $11.61(0.60)$ & $13.28(0.62)$ & 2.00 & 0.14 & \multirow{3}{*}{ Persistent $>$ Stopped**; Never $>$ Stopped* } \\
\hline PANSS positive $^{d}$ & 177 & $11.92(0.48)$ & $9.59(0.71)$ & $12.69(0.58)$ & 6.01 & $<0.01$ & \\
\hline PANSS general ${ }^{e}$ & 162 & $26.41(0.77)$ & $24.31(1.09)$ & $27.94(0.99)$ & 3.00 & 0.05 & \\
\hline $\mathrm{BDI}^{\mathrm{f}}$ mean (s.e.) & 162 & $11.13(1.09)$ & $10.46(1.75)$ & $15.97(1.73)$ & 3.26 & 0.04 & Persistent > Stopped*; Persistent > Never* \\
\hline Relapse, $^{g} \%$ & 181 & 35.6 & 32.9 & 56.3 & 3.23 & 0.04 & Persistent > Stopped*; Persistent $>$ Never* \\
\hline \multicolumn{8}{|c|}{ 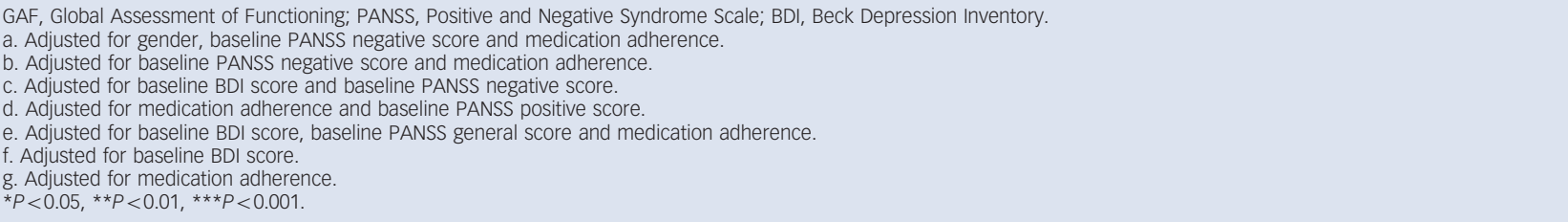 } \\
\hline
\end{tabular}

total and PANSS negative. The relapse rate in this group was $44.1 \%$ (45 of 102 participants) and this was also seen to vary significantly across substance misuse groups. Table 4 displays these results after adjusting for baseline confounding factors. Again, the pattern of results is similar to that for the whole sample, with significant differences between substance misuse groups in GAF total, GAF symptoms and PANSS positive. Differences in BDI score at 1 year seen in the whole sample were reflected in the schizophrenia group but did not reach significance. Relapse rates were again significantly higher in the persistent group compared with both the stopped $(P=0.01)$ and the never $(P=0.01)$ groups. There was no significant difference in relapse rates between the stopped and never groups.

\section{Discussion}

\section{Prevalence}

Prevalence rates of substance misuse from this study (baseline rates: any substance $43 \%$, alcohol $33 \%$, cannabis $21 \%$ ) are broadly comparable with rates observed in other UK first-episode samples, although such comparisons should be interpreted with caution because of different methodologies between studies. Different studies have used different inclusion criteria (particularly age range), different diagnostic criteria both in terms of substance misuse and first-episode psychosis and different methods of data collection (participant interview, informant or case notes), all of which may lead to variation in reported rates of prevalence.

In a Glasgow first-episode sample $(n=168)$, the rate of substance misuse at presentation was $37 \%$, with rates of drug and alcohol use of $19.5 \%$ and $11.7 \%$ respectively. The age range was 16-64, and included all individuals with psychotic symptoms. In that study, 'misuse' was defined as daily use for 2 weeks in the preceding year and information was gathered from participant interview; case notes were used only when participant interview was unavailable. ${ }^{2}$ In the West London First-Episode Schizophrenia Study $(n=152), 27 \%$ reported problem drinking and $35 \%$ reported substance use in the month prior to presentation. Participants included in this study were aged between 16 and 50, diagnosis was limited to DSM-IV schizophrenia or schizoaffective disorder, and substance misuse information was assessed at participant interview only. ${ }^{5}$ In the CAMEO specialist early intervention service in Cambridgeshire $(n=123)$, rates of lifetime DSM-IV

\begin{tabular}{|c|c|c|c|c|c|c|c|}
\hline \multirow[b]{2}{*}{ Adjusted 1-year score } & \multicolumn{4}{|c|}{ Substance misuse group } & \multirow[b]{2}{*}{$F$} & \multirow[b]{2}{*}{$P$} & \multirow[b]{2}{*}{ Significant pairwise comparisons } \\
\hline & $n$ & Never & Stopped & Persistent & & & \\
\hline \multicolumn{8}{|l|}{ GAF, mean (s.e.) } \\
\hline GAF total ${ }^{\mathrm{a}}$ & 97 & $56.50(2.06)$ & $59.77(3.30)$ & $44.27(3.84)$ & 5.24 & $<0.01$ & \multirow{3}{*}{$\begin{array}{l}\text { Persistent }<\text { Stopped }{ }^{* *} \text {; Persistent }<\text { Never }^{* *} \\
\text { Persistent }<\text { Stopped }^{* *}\end{array}$} \\
\hline GAF symptoms $^{b}$ & 96 & $57.31(2.24)$ & $63.16(2.98)$ & $49.86(3.05)$ & 5.22 & 0.02 & \\
\hline GAF disability ${ }^{c}$ & 93 & $54.17(2.26)$ & $59.26(3.37)$ & $50.81(2.78)$ & 1.87 & 0.16 & \\
\hline \multicolumn{8}{|l|}{ PANNS, mean (s.e.) } \\
\hline PANNS negative ${ }^{d}$ & 101 & $13.94(0.55)$ & $12.08(0.73)$ & $13.56(0.78)$ & 2.14 & 0.12 & \multirow{3}{*}{ Persistent $>$ Stopped**; Never > Stopped ${ }^{*}$} \\
\hline PANSS positive ${ }^{e}$ & 101 & $12.62(0.64)$ & $9.89(0.86)$ & $13.74(0.89)$ & 5.28 & $<0.01$ & \\
\hline PANSS general $^{f}$ & 97 & $27.28(0.96)$ & $24.51(1.42)$ & $28.00(1.21)$ & 1.93 & 0.15 & \\
\hline BDI,, mean (s.e.) & 92 & $11.18(1.47)$ & $10.16(2.00)$ & $16.30(2.03)$ & 2.72 & 0.07 & \\
\hline Relapse, \% & 102 & 38.0 & 33.3 & 68.0 & 4.11 & 0.02 & Persistent $>$ Stopped*; Persistent $>$ Never* \\
\hline \multicolumn{8}{|c|}{$\begin{array}{l}\text { GAF, Global Assessment of Functioning; PANSS, Positive and Negative Syndrome Scale; BDI, Beck Depression Inventory. } \\
\text { a. Adjusted for gender. } \\
\text { b. Adjusted for baseline PANSS negative score. } \\
\text { c. Adjusted medication adherence. } \\
\text { d. Adjusted for baseline PANSS negative score. } \\
\text { e. Adjusted for baseline PANSS positive score. } \\
\text { f. Adjusted baseline PANSS negative score, baseline PANSS general score and medication adherence. } \\
\text { g. Adjusted for baseline BDI score. } \\
\star P<0.05, * *<0.01 \text {. }\end{array}$} \\
\hline
\end{tabular}


misuse or dependence were found to be $43.1 \%$ for alcohol and $50.8 \%$ for cannabis. The sample included all people with a diagnosis of psychosis, information was gathered from clinical interview only and the age range for this sample was $17-35 .{ }^{8}$

Our study differs from these first-episode studies in that not only does it use information from clinical assessment but also it draws on available information from relatives and case notes to make a diagnosis of substance misuse, thus increasing the reliability of this particular measure. The large sample includes all incident cases of psychosis yet also allows for a reasonable subgroup analysis of schizophrenia-spectrum disorder. Furthermore, the use of a 12-month period of 'current' substance misuse makes the data both robust and inclusive.

We have also shown that prevalence rates of substance misuse fell in this cohort after 1 year to approximately half their previous value, which may suggest that routine psychiatric treatment may have some impact on rates of substance misuse early in the course of treatment. However, reduction in rates of substance misuse may also be attributable to other factors, including improved insight, lesser severity of illness, improved social support or reduced access to misused substances.

\section{Baseline characteristics}

There are two important observations to be made when looking at the baseline characteristics of this group. The first is between individuals with substance misuse and no substance misuse at baseline. People with substance misuse at baseline were more likely to be male and present at a younger age. In addition, this younger age at presentation was significantly associated with cannabis misuse in particular. This is in keeping with previous studies in this area, suggesting that male gender and substance misuse, particularly cannabis use, are associated with an earlier age at psychosis onset. ${ }^{2,3,5,7,9}$ although not all studies have found this association..$^{20}$ It should be noted that with respect to cannabis misuse and age at onset, we cannot determine direction of causality from this study, and other explanations for this association such as greater cannabis availability in younger peer networks and/or a greater propensity for younger individuals to misuse cannabis should also be considered as explanations for this association.

The second finding is within the group of participants with substance misuse at baseline. Those individuals who misused substances persistently over the first year appear to differ from those who stopped misusing substances after presentation in terms of their baseline characteristics. Individuals who are noted to have persistent substance misuse had more severe depressive symptoms at baseline than those who subsequently stopped using substances. The reason for this is not clear. It may be that the presence of more severe depressive symptoms at baseline makes people either less likely to respond to interventions that target substance misuse or more likely to self-medicate. A similar pattern for negative symptoms did not remain significant after adjusting for gender. Our findings are in keeping with other studies that have suggested that more severe negative symptoms at baseline are associated with male gender. ${ }^{9}$

Positive symptoms were also seen to vary significantly, with higher scores observed in the stopped group compared with the other two groups. Participants with higher symptom scores may be more likely to receive targeted substance misuse intervention from clinicians, may be more likely to experience enforced cessation through admission to hospital, greater disruption of social networks or both.

There was poorer medication adherence at 1 year among participants with persistent substance misuse. Medication adherence has previously been noted to be poor in this group, and is an important consideration given its association with substance misuse and poor outcome. ${ }^{21,22}$ This was considered as a confounder in this study, and our results show that the detrimental impact of persistent substance misuse on 1-year outcome is independent of medication adherence.

\section{Global assessment of functioning}

The GAF is a widely used outcome tool in first-episode psychosis studies. It has been shown to be reliable and correlated with concurrent ratings of symptoms and social functioning in the follow-up period rather than the acute psychotic episode. ${ }^{23} \mathrm{~A}$ limitation of this study is that GAF scores were not assessed at baseline, and this may influence interpretation of later scores.

However, studies examining the impact of substance misuse in first-episode psychosis have generally focused on clinical and symptomatic outcomes. Functional outcomes have only been included in a small number of studies. One small study $(n=58)$ found no impact of persistent substance misuse on social outcome, ${ }^{24}$ although poorer quality-of-life scores were seen in persistent substance misusers in a further group of people with early psychosis. ${ }^{25}$ A 15-month prospective follow-up study of 92 individuals with first-episode psychosis found heavy (but not mild) substance misuse to be associated with poorer functional outcome but not quality of life. ${ }^{13}$

In our study, the pattern of outcome was consistent between the total group and the subgroup of individuals with schizophrenia. There was also consistency across GAF subscales. There were no significant differences in outcome between the never and stopped groups, but the persistent group did significantly worse than both. The differences in outcome would also appear to be clinically significant with adjusted GAF scores differing by approximately 11 to 13 points between the stopped and persistent groups.

\section{Symptoms}

The substance misuse group to which an individual belongs appears to have an effect on two domains of symptoms - positive and depressive symptoms. There was no impact on negative symptoms or general psychopathology.

Participants with persistent substance misuse had more severe positive symptoms at 1 year than those who stopped misusing substances, despite having had less severe symptoms at baseline. Although clinically the differences were small, findings were in keeping with other studies that have examined this issue. People with first-episode psychosis and with persistent cannabis or substance misuse have been shown at 4 years to have more positive, but not negative symptoms than those who stopped using. ${ }^{12,13}$ Similarly, cannabis use has been shown to be associated with more severe positive, but not negative symptoms at 1,2 and 3 years after a first psychotic episode. ${ }^{25}$

In our study we also observed less severe positive symptoms at 1 year in the stopped compared with the never group despite the former group having had more severe symptoms at baseline. It may be that in people presenting with substance misuse at baseline, a greater proportion of the positive symptomatology is attributable to substance misuse such that relatively greater gains are achieved when this is stopped.

There was a clear impact of substance misuse on the severity of depression in our sample. This is evident at presentation and remains so at 1 year even after adjusting for depressive symptoms at baseline. The differences were clinically significant, and the fact that the pattern was still observed in the schizophrenia subgroup indicated that it is unlikely that the differences are accounted for by the presence of individuals with an affective disorder. There is 
some previous evidence to suggest that substance misuse, particularly cannabis misuse, in first-episode psychosis may increase the risk of depression. Although it has been suggested that cannabis misuse does not have an impact on affective scores in recent-onset schizophrenia, ${ }^{11}$ it has been shown in another firstepisode sample to be associated with increased scores for depression at 1 year, though not at 2 and 3 years. ${ }^{25}$ In a sample of older individuals with schizophrenia, current and lifetime comorbid substance misuse was associated with increased rates of clinically significant depression. ${ }^{26}$ It is not clear from our study whether the effect of substance misuse on depression is one of alcohol, cannabis or both, but clearly this is an area that needs further study.

\section{Relapse}

Our criteria for relapse were broad and included any relapse of symptoms following a period of remission as well as continuous illness over the first year. Nonetheless, our results were in keeping with other research in this area. Persistent substance misuse in the early course of first-episode psychosis has been shown to be associated with increased risk of readmission, ${ }^{6,24}$ increased risk of relapse $\mathrm{e}^{11,27}$ and a continuous course of illness. ${ }^{12,28}$

Importantly however, our study was able to show clearly that the increased relapse rate was limited to participants with persistent substance misuse and that the relapse rate in those who stopped misusing substances after presentation did not differ from the rate for those who had never misused substances.

\section{Strengths and limitations}

This study was epidemiologically robust and the investigators went to considerable effort to identify all new cases of psychosis presenting to psychiatric services over the 2-year period. The geographical area has a stable population with relatively low rates of immigration and emigration compared with the rest of the UK. ${ }^{29}$ The large sample is also a considerable strength of the study. It allows for a subgroup analysis of people with schizophrenia while at the same time maintaining reasonable numbers. The use of multiple sources to record substance misuse strengthens this particular measure. Incorporating a variety of baseline measures allows us to adjust for potential confounders, not least medication adherence, which is frequently associated with substance misuse and poor outcome.

Limitations include the lack of ability to precisely quantify drug use, although this may be difficult to measure accurately in practice. The use of only two time points for assessment meant we were unable to get a clear picture of the temporal relationship between substance misuse and clinical presentation over the first year. A significant number of individuals recruited to the study were lost to follow-up and although there was no evidence that they differed significantly at baseline, we cannot be sure that their exclusion has not biased the results of this study.

The use of OPCRIT to obtain a diagnosis does not allow for a specific category of 'drug-induced psychosis', as these individuals are categorised as having 'psychosis not otherwise specified'. It could be argued that the stopped group may contain an excess of patients with 'drug-induced psychosis', representing individual brief psychotic episodes that resolve on cessation of drug use and are associated with a better prognosis. Therefore the observed differences between the stopped and persistent groups may be attributable to differences in outcome between people with 'drug-induced psychosis' and 'schizophrenia'. However, analysis of the subgroup of individuals who meet diagnostic criteria for schizophrenia at 1 year mirrors the results for the group taken as a whole, indicating that the inclusion of participants with 'drug-induced psychosis' did not bias the results.

\section{Clinical implications}

This study confirms the high rates of substance misuse in people with first-episode psychosis observed in previous studies. It also highlights the association between persistent substance misuse and poor outcome in the first year after presentation. We were unable to determine from this study whether or not this association was causal. It is possible that individuals in the persistent group represented a subgroup of people with severe illness and poor prognosis who were more likely to misuse substances either as a feature of their illness or through selfmedication. However, when the results of this study are considered in the context of other research in this area, it clearly highlights the importance of addressing substance misuse early in people with first-episode psychosis.

The study clearly shows that substance misuse at presentation in first-episode psychosis is not in itself associated with adverse outcome. On all outcome measures, participants who stopped misusing substances did at least as well as those with no substance misuse.

For individuals who went on to misuse substances persistently, there were possible indicators from first presentation. In particular, these individuals tended to have less severe positive symptoms and more severe depressive symptoms. It may be that such a presentation makes substance misuse more difficult to detect, or less of a priority for intervention. Alternatively, these differences may represent a subtype of illness with a greater prevalence of depressive symptoms, increased likelihood of persistent substance misuse and poorer outcome.

The results suggest that substance misuse may already have been successfully targeted in this group to some extent. If this was the case, it may be reasonable to expect that enhanced detection and treatment of substance misuse in this population would bring about further gains in early outcome.

Identification and treatment of substance misuse should be a key component of early intervention services. This has implications for staff training and the structure of such services, which should focus on the recognition and assertive treatment of substance misuse in this patient group. However, further study is needed to determine whether this would result in greater reductions in rates of substance misuse, and whether or not reduced substance misuse is indeed associated with further improvements in outcome. Although addressing substance misuse in this area can often be challenging, it should be faced with optimism that early outcomes in this patient group can be significantly improved.

\footnotetext{
Aidan Turkington, MRCPsych, Ciaran C. Mulholland, MD, MRCPsych, Department of Psychiatry, Queen's University, Belfast; Teresa M. Rushe, PhD, Department of Of Psychiatry, Queen's University, Belfast; Teresa M. Rushe, PhD, Department of
Psychology, University of Ulster; Rick Anderson, MRCPsych, Rosalind McCaul, BSC, Suzanne L. Barrett, PhD, Ruth S. Barr, MD, MRCPsych, Stephen J. Cooper, MD, FRCPsych, Department of Psychiatry, Queen's University, Belfast, UK

Correspondence: Aidan Turkington, Department of Psychiatry, Whitla Medical Building, 97 Lisburn Road, Belfast BT9 7BL, UK.

Email: aidant@doctors.org.uk

First received 21 Jul 2008, final submission 15 Dec 2008, accepted 5 Feb 2009
}

\section{Funding}

Funded by the Research and Development Office of the Northern Ireland Health and Personal Social Services.

\section{Acknowledgements}

We thank the medical and nursing staff of the trusts involved, and Dr Rachel Smylie and Dr Mark Finnerty for their assistance. We also thank the AESOP (Aetiology and Ethnicity 
in Schizophrenia and Other Psychoses) study investigators for permission to use and publish the Record of Substance Abuse schedule.

\section{References}

1 Regier DA, Farmer ME, Rae DS, Locke BZ, Keith SJ, Judd LL, et al. Comorbidity of mental disorders with alcohol and other drug abuse. Results from the Epidemiologic Catchment Area (ECA) Study. JAMA 1990; 264: 2511-8.

2 Cantwell R, Brewin J, Glazebrook C, Dalkin T, Fox R, Medley I, et al. Prevalence of substance misuse in first-episode psychosis. $\mathrm{Br} J$ Psychiatry 1999: 174: 150-3.

3 Van Mastrigt S, Addington J, Addington D. Substance misuse at presentation to an early psychosis program. Soc Psychiatry Psychiatr Epidemiol 2004; 39 69-72.

4 Lambert M, Conus P, Lubman DI, Wade D, Yuen H, Moritz S, et al. The impac of substance use disorders on clinical outcome in 643 patients with firstepisode psychosis. Acta Psychiatr Scand 2005; 112: 141-8.

5 Barnes TRE, Mutsatsa SH, Hutton SB, Watt HC, Joyce EM. Comorbid substance use and age at onset of schizophrenia. Br J Psychiatry 2006; 188 237-42.

6 Wade D, Harrigan S, Edwards J, Burgess PM, Whelan G, McGorry PD. Substance misuse in first-episode psychosis: 15-month prospective follow-up study. Br J Psychiatry 2006; 189: 229-34.

7 Larsen TK, Melle I, Auestad B, Friis S, Haahr U, Johannessen JO, et al. Substance abuse in first-episode non-affective psychosis. Schizophr Res 2006; 88: 55-62.

8 Barnett JH, Werners U, Secher SM, Hill KE, Brazil R, Masson K, et al. Substance use in a population-based clinic sample of people with firstepisode psychosis. Br J Psychiatry 2007; 190: 515-20.

9 Veen ND, Selten JP, van der Tweel I, Feller WG, Hoek HW, Kahn RS., Cannabis use and age at onset of schizophrenia. Am J Psychiatry 2004; 161: 501-6.

10 Addington J, Addington $\mathrm{D}$. Effect of substance misuse in early psychosis. $\mathrm{Br} \mathrm{J}$ Psychiatry 1998; 172 (suppl 33): s134-6.

11 Linszen DH, Dingemans PM, Lenior ME. Cannabis abuse and the course of recent-onset schizophrenic disorders. Arch Gen Psychiatry 1994; 51: 273-9.

12 Grech A, Van Os J, Jones PB, Lewis SW, Murray RM. Cannabis use and outcome of recent onset psychosis. Eur Psychiatry 2005; 20: 349-53.

13 Wade D, Harrigan S, McGorry P, Burgess PM, Whelan G. Impact of severity of substance use disorder on symptomatic and functional outcome in young individuals with first-episode psychosis. J Clin Psychiatry 2007; 68: 767-74

14 Kay SR, Fiszbein A, Opler LA. The positive and negative syndrome scale (PANSS) for schizophrenia. Schizophr Bull 1987; 13: 261-76.

15 Beck AT, Ward $\mathrm{CH}$, Mendelson M, Mock J, Erbaugh J. An inventory for measuring depression. Arch Gen Psychiatry 1961; 4: 561-71.
16 Jablensky A, Sartorius N, Ernberg G, Anker M, Korten A, Cooper JE, et al. Schizophrenia: manifestations, incidence and course in different cultures. A World Health Organization ten-country study. Psychol Med Monogr Supp I 1992; 20: 1-97.

17 Endicott J, Spitzer RL, Fleiss JL, Cohen J. The Global Assessment Scale. A procedure for measuring overall severity of psychiatric disturbance. Arch Gen Psychiatry 1976; 33: 766-71.

18 McGuffin P, Farmer A, Harvey I. A polydiagnostic application of operationa criteria in studies of psychotic illness. Development and reliability of the OPCRIT system. Arch Gen Psychiatry 1991; 48: 764-70.

19 World Health Organization. The ICD-10 Classification of Mental and Behavioural Disorders: Clinical Descriptions and Diagnostic Guidelines. WHO, 1992.

20 Sevy S, Robinson DG, Holloway S, Alvir JM, Woerner MG, Bilder R, et al. Correlates of substance misuse in patients with first-episode schizophrenia and schizoaffective disorder. Acta Psychiatr Scand 2001; 104: 367-74.

21 Verdoux H, Lengronne J, Liraud F, Gonzales B, Assens F, Abalan F, et al. Medication adherence in psychosis: predictors and impact on outcome. A 2-year follow-up of first-admitted subjects. Acta Psychiatr Scand 2000; 102: 203-10.

22 Coldham EL, Addington J, Addington D. Medication adherence of individuals with a first episode of psychosis. Acta Psychiatr Scand 2002; 106: 286-90.

23 Startup M, Jackson MC, Bendix S. The concurrent validity of the Global Assessment of Functioning (GAF). Br J Clin Psychol 2002; 41: 417-22.

24 Sorbara F, Liraud F, Assens F, Abalan F, verdoux $H$. Substance use and the course of early psychosis: a 2-year follow-up of first-admitted subjects. Eur Psychiatry 2003; 18: 133-6.

25 Addington J, Addington D. Patterns, predictors and impact of substance use in early psychosis: a longitudinal study. Acta Psychiatr Scand 2007; 115: $304-9$.

26 Margolese HC, Carlos Negrete J, Tempier R, Gill K. A 12-month prospective follow-up study of patients with schizophrenia-spectrum disorders and substance abuse: changes in psychiatric symptoms and substance use. Schizophr Res. 2006; 83: 65-75.

27 Malla A, Norman R, Bechard-Evans L, Schmitz N, Manchanda R, Cassidy C. Factors influencing relapse during a 2-year follow-up of first-episode psychosis in a specialized early intervention service. Psychol Med 2008; 21: $1-9$

28 Manchanda R, Norman RM, Malla AK, Harricharan R, Northcott S. Persistent psychoses in first episode patients. Schizophr Res 2005; 80: 113-6.

29 Dunnell K. The changing demographic picture of the UK: national statistician's annual article on the population. Popul Trends 2007; 130 9-21. 